\title{
NEUROPHYSIOLOGICAL CONTROL OF SLEEP WITH SPECIAL EMPHASIS ON MELATONIN
}

\author{
Iv. Penchev Georgiev* \\ Department of Pharmacology Animal Physiology and Physiological Chemistry, \\ Faculty of Veterinary Medicine, Trakia University, Stara Zagora, Bulgaria
}

\begin{abstract}
Sleep and wakefulness are two main types of human and animal behavior. On the average human beings spend about one-third of their lives asleep. The sleep-wake cycle is the most important circadian rhythms which alternates in a periodic manner lasting for about 24 hours. Sleep is determined as the natural periodic suspension of consciousness characterized by relative immobility and reduced responsiveness to external stimuli. The researchers have found and identified many special brain structures and systems controlling waking, rapid eye movement (REM) sleep and nonrapid eye (NREM) sleep and the transitions among these states.

Currently, there is an enhanced interest of researchers toward sleep and its neurophysiological mechanisms of regulation because the number of people suffering from various sleep disturbance such as insomnia, delayed sleep onset, duration and propensity of sleep, worldwide dramatically increases. In addition to the next day drowsiness, nervousness, tiredness and decreased workability, it has been suggested that sleep is important also for the maintaining of mood, memory and cognitive function of the brain and is essential for the normal functioning of the endocrine and immune systems. More recently, new studies show a sustained link between sleep disorders and different serious health problems, including obesity, insulin resistance, type 2 diabetes mellitus, cardio-vascular diseases and depression.

Therefore, the purpose of this review is to summarize and analyze the available data about the neurological control of wakefulness, non-rapid-eye-movement (NREM) sleep and rapid- eyemovement (REM) sleep creating a substantial basis for better understanding different sleep disorders. Special attention is paid on the pharmacological aspects and use of some new classes of sleep promoting agents - melatonin, melatonin receptor agonists and orexin receptor antagonists.
\end{abstract}

Key words: sleep, NREM sleep, REM sleep, circadian regulation of sleep, homeostatic regulation of sleep, melatonin, melatonin receptor agonists, orexin receptor antagonists

\section{INTRODUCTION}

Sleep and wakefulness are two hallmark characteristics of human and animal behavior. On average human beings spend about onethird of their lives asleep. The sleep-wake cycle is the most important circadian rhythm which alternates in a periodic manner lasting for about 24 hours. Sleep is determined as the

\footnotetext{
*Correspondence to: Prof. Dr. Ivan Penchev Georgiev, Dsc., Department of Pharmacology Animal Physiology and Physiological Chemistry, Section of Anima Physiology, Trakia University, Faculty of Veterinary Medicine, 6000 Stara Zagora, Bulgaria, Phone: +359 42 887064791; +35942699629, E mail: iv_p63@abv.bg / ivanpenchev@uni-sz.bg
}

natural periodic suspension of consciousness characterized by relative immobility and reduced responsiveness to external stimuli (1). In contrast, the waking state is characterized by purposeful motor activity and increased ability to adequate responsiveness to various environmental stimuli. The disorders in sleepwake cycle can cause detrimental abnormalities in the organism as many other physiological processes are closely associated with this cycle.

Evolutionary, a form of non-rapid-eyemovement (NREM) sleep is found in most animals and argued the fact that sleep is an essential vital function of the organisms. 
Rapid-eye-movement (REM) sleep appears later in the evolution - in a primitive form in reptiles and bird and in a true mature form in mammals (2). It was long time thought that sleep is a passive and relatively constant process of "switch off" of most of the bodily functions, while our body "switch back on" when we awake. In other words, till the last century sleep was mainly defined as a consequence of reduced sensory input and low brain activity (3). Therefore, the simplest explanation of sleep is the need to recover and repair the cellular metabolism and the neuronal networks and brain functions after the active period of wakefulness. In fact, it was found that sleep is a very active state of the brain during which many vital physiological processes occur $(4,5)$. In addition, the rapid development of neuroscience, neurophysiology and electrophysiology, have clearly indicated that during sleep the brain activity is even more varied and more complex than during the waking state. It is important to note, however, that there is a marked decrease in the perception due to the rise of the threshold for both external and internal stimuli (3).

Despite the purpose and the need of sleep is one of the biggest mysteries in biology, over the last $10-15$ years great progress has been made in understanding the neuronal mechanisms that control sleep-wake cycle (6$8,4,2,9,5)$. The researchers have found and identified many special brain structures and systems controlling the occurrence and timing of wake and both phases of sleep - rapid eye movement (REM) sleep and - REM (NREM) sleep and the transitions between them as well $(9,3)$.

Currently, there is an enhanced interest of researchers toward sleep and its neurophysiological mechanisms of regulation because the number of people suffering from various sleep problems such as insomnia, delayed sleep onset, duration and the propensity of sleep, worldwide dramatically increases $(8 ; 10)$. In addition to the next day drowsiness, nervousness, tiredness and decreased workability, new data reveal that sleep is essential for the maintaining of mood, memory and cognitive capacity of the brain (11-15). It is also involved in the control of the normal functions of the endocrine system and immunity. More recently, new studies show a sustained link between sleep disorders and different serious health problems, including obesity, insulin resistance, type 2 diabetes mellitus, cardio-vascular diseases and depression (16-19).

Despite sleep disorders affect humans of all ages, they are more pronounced in adults. Abnormal sleep can lead to dysfunction of other organs and physiological systems and/or disturbance in brain functions and neuropsychiatric disorders in particular. Therefore, better understanding the physiology of sleep is crucial in the management of different abnormalities like insomnia, attention deficit and inability to concentrate, depression, and obesity related diseases.

Therefore, the purpose of this review is to summarize and analyze the available data about the neurological control of wakefulness, non-rapid-eye-movement (NREM) sleep and rapid- eye-movement (REM) sleep creating a substantial basis for better understanding different sleep disorders. Special attention is paid on the pharmacological aspects and use of some new classes of sleep promoting agents melatonin, melatonin receptor agonists and orexin receptor antagonists.

\section{Types of sleep}

Despite sleep is an unconscious state characterized by decreased responsiveness, in fact, it is an active physiological process, as all organs and regulatory systems continue to function.

The current classification of sleep types and stages was done in 2004 by the American Academy of Sleep Medicine (20, 21). Accordingly, sleep was categorized into 2 distinct types:

1.Non-rapid eye movement sleep (Non- REM; NREM). Based on the EEG pattern it is also called "slow wave sleep" (SWS) and is further divided into 4 stages: Stage 1; Stage 2; Stage 3 and Stage 4.

2. Rapid eye movement (REM) sleep. Changes in brain activity that take place during the different type and stages of sleep are measured using an electro encephalogram (EEG). Electroencephalography is a useful method for the recording of small voltage alterations on the surface of the scalp due to the summed activity of neurons in a separate region of brain cortex. As such on the EEG we can detect the increased or decreased activity of these regions during waking state and/or during different sleep stages. 
It is important to note that electroencephalogram and brain waves markedly differs among types and stages of sleep and are the basis for such divisions of sleep.

\section{Brain waves}

Electrophysiological studies show that in humans five main different types of electrical patterns are detected $(20,22,21,1)$. They can be recorded and observed as brain waves on the electroencephalogram (EEG). Actually, electroencephalography is a tool to measure the activity of different regions of the brain cortex by detecting the bioelectrical events i.e. action potentials as small voltage changes on the surface of the scalp. Each brain wave has a specific purpose and is depending on the functional activity of the brain during the waking and sleeping periods $(22,1)$. If one of the brain waves is over- or under expressed, it is usually a result of some functional disorders in some brain regions. It is important to point out that there is no brain wave which is more or less important than the others. Therefore, when we refer to a certain brain wave, this means that a particular brain wave is dominant among others. During waking state throughout the day and during the sleeping period throughout the night the EEG will display all 5 types of brain waves at the same time $(22,2)$. However, one particular brain wave will dominate depending on the state of consciousness that we are in.

The brain waves have at least 2 important characteristics on EEG, which differ in each wave: frequency and amplitude. The frequency is measured in $\mathrm{Hz}$ while the amplitude is measured in $\mu \mathrm{V}$. The frequency and amplitude are in a close inverse interrelation i.e. high frequency/low amplitude and high amplitude/low frequency. The brain waves in order of highest to lowest frequency are as follows: gamma, beta, alpha, theta, and delta. For more details concerning the characteristic features of each brain wave please see $(21,23$, 1) (Figure 1):

\section{Normal Adult Brain Waves}

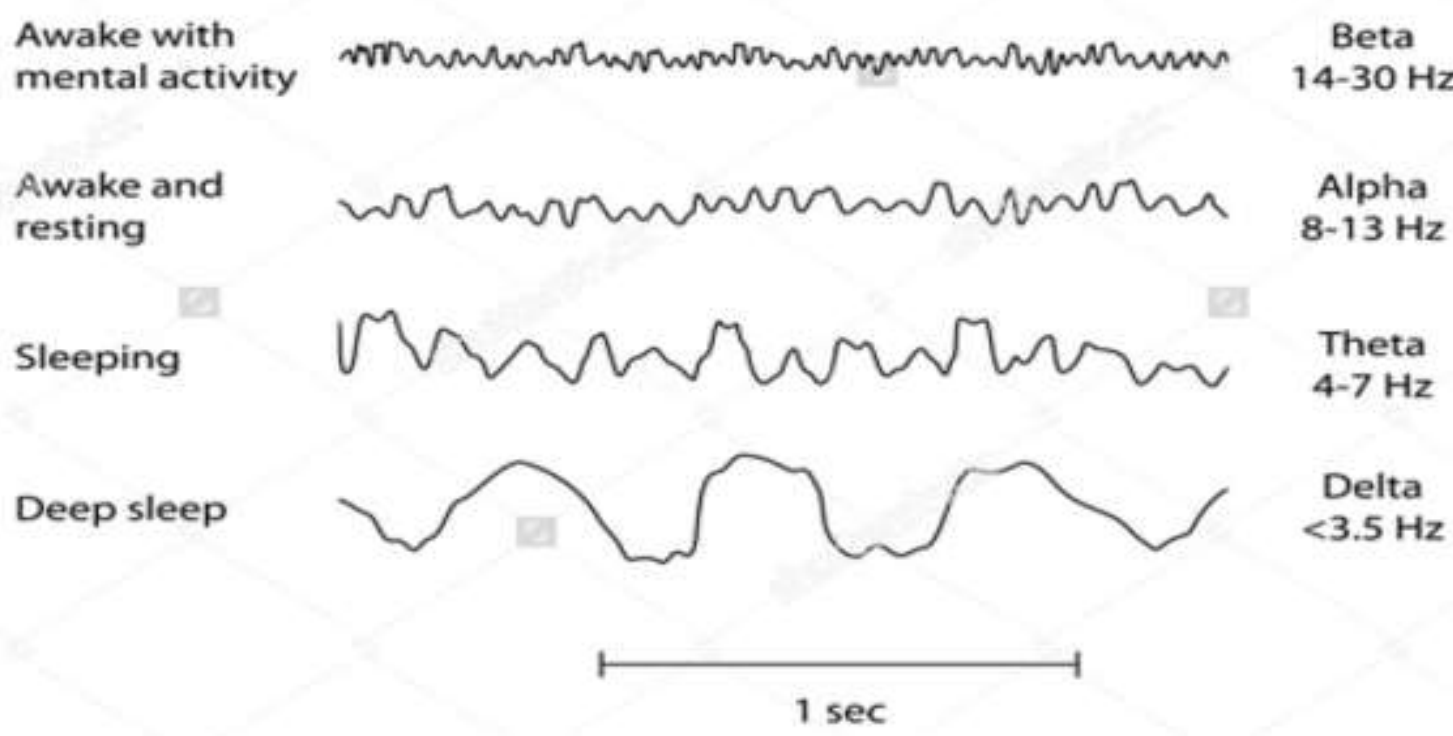

\section{shutterst,sck}

Figure 1. Normal brain vawes in adult humans (from: https://www.bing.com/images/)

1. Gamma waves - frequency range: $40 \mathrm{~Hz}$ to $100 \mathrm{~Hz}$ (fast wave - very low amplitude and very high frequency). Optimal gamma waves are indicators for binding senses and perception, cognition, information processing, learning and REM sleep; too much: -anxiety, stress; too little - depression, learning disabilities. Meditation is found to increase gamma waves. Usually, in clinical practice gamma waves can not to be registered with the conventional equipment.

2. Beta waves - frequency range: $12 \mathrm{~Hz}$ to $40 \mathrm{~Hz}$ (fast wave - low amplitude and high 
frequency). Beta rhythm is characterized with high frequency low amplitude brain waves, which are mainly registered while we are awake. They are involved in logical thinking, writing, and reading. In other words beta waves characterize our everyday activity. Optimal beta waves conscious focus, memory, problem solving. Coffee, energy drinks and other stimulants markedly increase beta waves.

3. Alpha waves - Frequency range: $8 \mathrm{~Hz}$ to $12 \mathrm{~Hz}$ (moderate frequency). Alpha rhythm is between beta (high) and theta (low) rhythm. It is recorded when the eyes are closed i.e. first stage of NREM sleep (see later). Too much alpha wave: daydreaming, inability to concentrate and to focus on; too little - anxiety, big stress, insomnia; optimal - complete relaxation. The following substances have been found to increase alpha rhythm: alcohol, marijuana and some antidepressants

4. Theta waves - Frequency range: $4 \mathrm{~Hz}$ to $8 \mathrm{~Hz}$ (slow wave - high amplitude and low frequency). Theta waves are involved in daydreaming and sleep. Too much theta waves are indicators of deep relaxation and hypnotic state. It is also recorded during restorative sleep - second and third stage of NREM sleep (see later). Hypnotic drugs and other relaxants such as benzodiazepines and neuroleptics may increase theta waves.

5. Delta waves - frequency range: $0 \mathrm{~Hz}$ to $4 \mathrm{~Hz}$ (slow wave - very high amplitude and very low frequency). They are hallmark of the deepest and restorative sleep. Depressant drugs and sleep increase delta waves.

During the waking state when we are with open eyes and our mind is focused, usually on EEG dominate low amplitude high frequency waves (beta rhythm). This pattern of EEGis also known as desynchronized because many neurons are firing independently. Similar desynchronized pattern of EEG is recorded during the REM sleep which is also called paradoxical sleep. In contrast, during the NREM sleep the EEG pattern is synchronized dominated by larger amplitude, lower frequency waves (alpha, teta, delta rhythm) (22). As much are the waves with high frequency and low amplitude (beta waves in active wake state and REM sleep) as greater is the brain activity. In contrast, as much are the waves with high amplitude and low frequency as lower is the brain activity (alpha, teta and especially delta waves during deep sleep stage 3 and 4 of NREM sleep) (see next chapter).

\section{Non-rapid-eye-movement (NREM) sleep} NREM sleep is characterized by a reduction in physiological activity. As sleep gets deeper, the brain waves as measured by EEG get slower and have greater amplitude, breathing and heart rate slow down, and blood pressure drops. NREM sleep is known also as synchronized or quiet sleep. The NREM phase consists of four stages as described in details $(20,22,21,24,1)$ :

* Stage 1 NREM sleep (Fig. 2): Stage 1 is a time of drowsiness or transition period between wakefulness and sleep. Eyes are closed and slow rolling eye movements $(0.25-0.5 \mathrm{~Hz})$ are present, but they disappear in stage 2 and deeper sleep stages (stage 3 and 4). There is a decrease in the heart and respiratory rates. In addition, a marked reduction in muscle tone and body temperature are recorded. The brain wave activity is dominated by alpha and theta rhythm. Alpha waves are recorded in the early phase of stage 1 which are with low frequency $(8-13 \mathrm{~Hz})$ and high amplitude and become synchronized. Further in stage 1 sleep, there is a lowing down of alpha rhythm and an increase in theta waves activity. Theta waves are characterized by lower frequency $(4-7 \mathrm{~Hz})$ and higher amplitude than alpha waves. In this stage, it is easy to awake and if so, people even say that they have not been asleep.

* Stage 2 NREM sleep (Figure 2): Stage 2 sleep the light sleep period during which eye movement stop and the body goes into a state of almost complete relaxation. It is the relatively largest period of a normal sleep during the night. As an individual moves into stage 2 sleep, the pattern of the brain activity is dominated by theta waves $(4-8 \mathrm{~Hz})$ which are interrupted by short bursts of in ceased frequency waves known as sleep spindles. Actually, the sleep spindle is a rapid burst of high frequency brain waves $(12-16 \mathrm{~Hz})$ that are probably important for learning and memory (25-28). In addition, the appearance of K-complexes is often associated with stage 2 sleep. Kcomplexes are transient diphasic waves 
with high amplitude pattern (>100) of brain activity and last more than $200 \mathrm{~ms}$. Therefore, the distinctive elements of EEG characterizing stage 2 sleep is the appearance of sleep spindles and/or Kcomplexes. The presence of sleep spindles however, is enough to define stage 2 sleep because K-complexes are typically associated with a spindle. The heart rate and body temperature continue to decrease.

- Stage 3 and 4 (Figure 2): Stages 3 and 4 (referred to usually together as stage 3 or as slow wave deep sleep), are characterized by the domination of high amplitude and low frequency $(0-4 \mathrm{~Hz})$ slow brain waves called delta waves. Because the difference between both stages is quite small usually they are presented together. It is generally accepted however, that in stage 4 delta waves dominate and are more than $50 \%$. Blood pressure and body temperature fall, breathing frequency slows. Sleep is deeper, with no eye movement and decreased muscle activity. This is the period when the body repairs muscles and tissues, stimulates growth and development, boosts immune function, and produces and stores the energy needed for for the next day activities. It is much more difficult to be awakened during these stages than during earlier stages. Individuals who are awakened report that they not feel refreshed even more they feel tired and disoriented $(29,30)$. Sleep walking or night terrors, experienced by some people also occur in these stages.

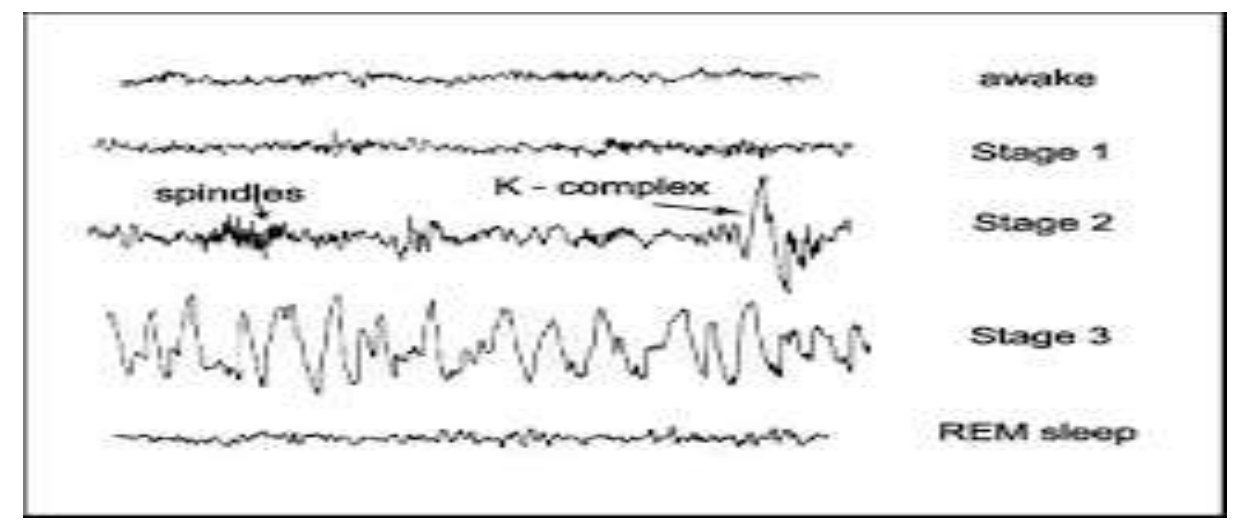

Figure 2. Normal brain waves during different sleep types and stages (from: $\underline{\text { https://www.bing.com/images/) }}$

\section{Rapid-eye-movement (REM) sleep}

The rapid eye movement (REM) sleep was firstly discovered in 1953. Since that period three basic states of behavior have been defined: wakefulness, rapid eye movement (REM) sleep, and non-rapid eye movement (NREM) sleep. $(22,21,31,1)$. The REM phase of sleep is established in mammals and to a lesser extends in reptiles and birds while in other animals its presence is less studied (2).

REM sleep is an active phase of sleep characterized by intense brain activity. Physiological mechanisms of REM sleep and its importance for health are not well studied. The complex relationships among different groups of neurons involved in the regulation of the main characteristics of REM sleep are not completely elucidated. The name REM sleep comes from the presence of one of the most important feature - rapid conjugate eye movements in different directions i.e. rapid eyes movements $(22,21,9)$.

The onset of the first REM sleep period occurs about 70 minutes after initially falling asleep. Normally, in adult people, four to six REM periods occur each night depending on the duration of the total sleep time. During this final phase of each sleep cycle, the brain becomes more active. It is thought that REM sleep plays an essential role in learning and memory function, because the brain consolidates and processes information from the day so that it can be stored in long-term memory $(32,22,33)$.

Despite a person is deeply asleep during REM sleep EEG shows that some brain areas, including brain cortex, are in a very active state comparable to that during waking state $(21,34,1)$ (Figure 2). For this reason, REM sleep is also known as paradoxical, desynchronized or active sleep since the EEG 
has almost the same characteristics as during waking state i.e. dominated by highfrequency, low-amplitude desynchronized brain waves (beta waves) despite the level of consciousness is very limited. In addition to rapid eye movements, other hallmarks of REM sleep include: almost complete lack of skeletal muscle tone (atonia) leading to paralysis, with the exception of extraocular muscles responsible for eyes movement and muscles involved in respiration - diaphragm and intercostal muscles); penile erection $(35-38,9)$. Breathing becomes more rapid, irregular and heart rate and blood pressure increase. This is also the sleep stage in which most dreams occur. $(36,37)$.

The REM sleep is completely different from all 4 phases of non-REM sleep. Therefore, this is an unique phase of sleep which is regulated in a different way and by different neurological mechanisms анд браин струцтурес as compared with NREM sleep. Despite there is a disagreement within the scientific community about the relative importanceof NREM and REM sleep for the cognition and memory, it is generally accepted that similar to NREM sleep, REM sleep and more specifically, the right balance between both phases of sleep, is involved in Txe processes of learning, memory, mood and ability to concentrate (39-42). It is also important to note that both types of sleep (NREM and REM) function independently and cannot replace and compensate each other (41). For example, if an individual is deprived of REM sleep and then allowed to sleep, he will spend more time in REM sleep. (41). This phenomenon is known as the REM rebound, and suggests that REM sleep, similar to NREM sleep, is probably also under homeostatic regulation.

\section{Sleep Architecture}

Based on the experimental data from sleep research it has been established that the approximate needed duration of sleep is depending on the age. The adults need on the average a range of 7 to 9 hours of sleep; teenagers need about 9 hours, and infants around 16 hours per day. For the normal restorative sleep however, the right combination of NREM and REM sleep during the night is of primary importance. As such, in normal sleep, NREM and REM sleep must alternate throughout the night and this pattern is called "sleep architecture" $(43,23)$. During a night an individual spends about $75-80 \%$ of total sleeping period in NREM phase of sleep and $20-25 \%$ - in REM sleep $(36,23)$.

Normally, during the night 4 to 6 sleep cycles occur. A full sleep cycle is composed of NREM and REM sleep that alternate every 90 to 110 minutes. The time course of different types and stages of sleep and their approximate length during the night could be presented on a special graphic called hypnogram as shown in Figure 3.

The length of the first sleep cycle is usually about 90 minutes with the following cycles averaging around 100-120 minutes and being shorter in children. Each cycle starts and follows the stages of non-REM sleep (stage 1 stage 2 - stage 3/4) and back through the stages (stage 3/4 - stage 2 - stage 1) (Figure 3). Then, instead of waking, starts a short period of REM sleep, and thereafter going back through the stages (stage 1 - stage 2 and stage 3/4) in a new cycle (see the hypnogram). Except the first cycle, after each REM phase there are typically very short periods of waking (lasting several seconds) but usually they are not essential and do not break down the sleep. During the night, the duration of stage 3/4 (deep sleep) decreases while the length of REM sleep periods increases. As a result, the proportion of stage 3 sleep is a greater early in the night, and the proportion of REM sleep is greater later in the night, especially during the last two sleep cycles close to the morning. In the morning time, all sleep consists almost of stages 1, 2 of NREM sleep and REM sleep (Figure 3). Usually, adults spend almost half of total sleep time in stage 2 of NREM sleep, about 20 percent in REM sleep, and the remaining time in the other stages (Figure 3). The duration in any given stage however, is not constant over the course of a night and may vary among individuals.

In addition to these changes in time course of the night, the sleep architecture varies also with age. Normal adults spend 20-25 percent of sleep time in REM, while in newborn babies the length of REM sleep is about half their sleep time. In young children deep NREM sleep (stages 3 and 4) dominate. With advance age, however, the amount of stages 3 and 4 of NREM sleep decreases accompanied with an increase of the lighter sleep periods i.e. stages 1 and 2. It is important to outline that despite sleep become more fragile in older people, the need for sleep does not decrease with age. 


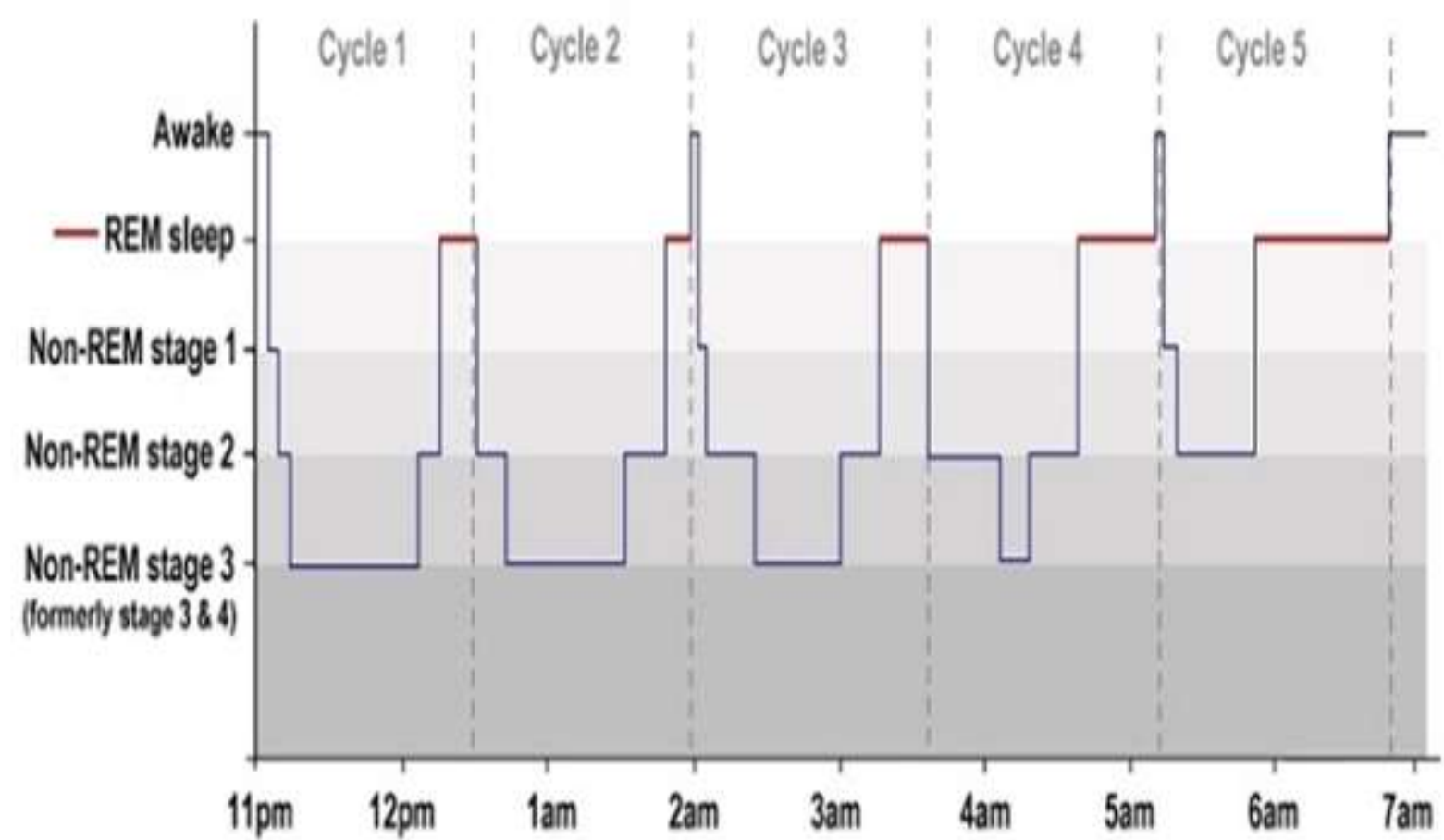

Figure 3. Normal hypnogram during different sleep stages and sleep cycles during the night (from: https://www.bing.com/images/)

\section{Neuronal networks regulating wakefulness}

About 90 years ago von Economo suggested that wakefulness is caused by an ascending arousal system originating from the brainstem (8). Consequently, all contemporary theories are based mainly on this hypothesis. Indeed, it has been experimentally proven that such system exists, located in a special brain structure - reticular formation (RF). It was called ascending reticular activating system (ARAS) which plays a pivotal role in the regulation of sleep-wake cycle. The ARAS extends from the upper part of medulla oblongata passing through the pons and midbrain up to diencephalon (thalamus and hypothalamus). It is predominantly composed of loosely clustered neurons and is crossed by a network of long bundles of ascending and descending fibers $(22,8,23)$. On the level of diencephalon ARAS separates into two branches - dorsal and ventral $(7,2)$. The dorsal pathway of the ARAS comprises glutamatergic neurons of reticular formation in midbrain, pons and medulla oblongata and originates from cholinergic neurons in the pedunculopontine tegmental (PPT) and later dorsal tegmental (LDT) nuclei in brainstem. The cholinergic axons of this branch of ARAS innervates and activates neurons in the specific relay thalamic nuclei, responsible for the transmission of impulses toward corresponding cortical areas - vision area (occipital lobe), auditory and equilibrium areas (temporal lobe), sensory area (parietal lobe - sensation from skin and muscles). The neurons of the PPT and LDT are very active during wakefulness and rapid eye movement sleep (REM sleep) and are almost inactive during slow wave sleep, known also as non-REM sleep when the activity of the brain cortex is markedly diminished $(7,2,23)$.

The axons of the ventral branch of the ARAS originate from a number of monoaminergic neurons in brain stem comprising noradrenergic in locus coerulus (LC), dopaminergic in ventral tegmental area (VTA), serotoninergic dorsal and median raphe nuclei in brain stem, glutamatergic in parabrachial nucleus $(23,2)$. They synapse onto glutamatergic, histaminergic and orexinergic neurons in the posterior/lateral hypothalamus and converge and contact with caudal basal forebrain cholinergic, GABA-ergeticand glutamatergic neurons. Axons of the cholinergic caudal basal forebrain neurons project directly and activate cortex (2). Recently it has been found that orexin (hypocretin) containing efferent neurons in lateral hypothalamus activates brain cortex in 
two ways: directly, and indirectly, by activating almost all above mentioned components of the ARAS $(23,2)$. The monoaminergic and orexinergic neurons are most active during wakefulness and less active during slow wave and REM sleep, while basal forebrain cholinergic neurons are equally active during wakefulness and REM sleep. Therefore, the waking state is caused and maintained by orchestrated and coordinated activity of the monoaminergic and cholinergic neurons included in the two branches of the ARAS and orexigenic neurons in lateral hypothalamus.

\section{Regulation of non-rapid-eye-movement sleep (non-REM sleep; NREM sleep)}

Because the main characteristics of NREM sleep on EEG are high amplitude-low frequency waves $(4-8 \mathrm{~Hz}$ theta waves and 1-4 $\mathrm{Hz}$ delta waves) this types of sleep is also known as slow wave sleep (SWS). It is important to note that there is no nerve centers or nuclei that directly induce sleep, in other words there is no sleeping center in the brain. However, it has been found that in anterior hypothalamus there is a special population of neurons included into ventrolateral preoptic nucleus (VLPO) which are strongly active during non-REM sleep $(22,8,23,44)$. The efferent fibers of VLPO release the main inhibitory neurotransmitters in the brain gama-aminobutiric acid (GABA) and galanin. The efferents of the VLPO connect with some of the nuclei of the ARAS such as the noradrenergic LC in brainstem and also the histaminergic TMN in posterior hypothalamus which are well known to stimulate the activity of brain cortex directly and indirectly, via connections with cholinergic PPT and BF nuclei (LC) $(23$; 44). As such, VLPO markedly decreases the activity of the arousal system during non-REM sleep. During wakefulness however, afferent noradrenergic nerve fibers from LC inhibits VLPO neurons via binding to alpha-2 noradrenergic receptors. Hence, it was suggested that there are reciprocal inhibitory interactions between arousal and sleep promoting neurons $(6,22,9,23,44)$. As a result, the activity of VLPO is down-regulated during waking state by some of the monoaminergic components of the arousal system it blocks during sleep which is an important neurological mechanism regulating sleep-wake cycle in mammals. Such reciprocal bidirectional interactions among waking- and sleep-inducing nuclei acting on the basis of the "flip-flop" or "on-off" model ensure rapid change between sleep and waking state (23, 44). This model may, therefore explain the very short sleep-wake transition periods (taking about $1 \%$ to $2 \%$ of the wake-sleep cycle).

Despite many debates on explaining the need for sleep, it is generally accepted that the main purpose of sleep is the restoration of the equilibrium in body functions which is disturbed to some extend during wakefulness. Therefore, sleep is an important component of the homeostasis but the underlying mechanisms regulating sleep however, remain uncertain. Therefore, the real debate is on the elucidation of the key mechanisms regulating the onset and maintenance of sleep behavior and the change between sleep and arousal state. A strong dependence between the changes of light-dark and wake-sleep cycle is evident. In this sense two separate mechanisms of regulation of sleep have been proposed: homeostatic (called process $\mathrm{S}$ ) and circadian (called process C) drives for sleep, respectively $(45,22,23)$. The timing of sleep is regulated by the circadian pacemaker (process $\mathrm{C}$ ), located in the hypothalamic nucleus (SCN) (46). The depth of sleep is however, regulated homeostatic ally (process S), with increasing sleep propensity during waking and decreasing of sleep pressure during sleep (47).Prolonged waking is compensated by deeper and prolonged sleep. The homeostatic sleep process is registered on electroencephalogram (EEG) as slow-wave activity (SWA; EEG is dominated by delta waves with high amplitude and low frequency - below $5 \mathrm{~Hz}$ ) during nonrapid eye-movement (NREM) sleep as a result of higher homeostatic sleep pressure $(48,49)$

\section{Homeostatic regulation of NREM sleep}

The assumption that there is a homeostatic element in sleep control comes from the observation that sleep deprivation is followed by an extra compensatory period of sleep the duration of which is proportional to the period of deprivation. It has been found that the two types of sleep, NREM and REM sleep, have different homeostatic mechanisms of regulation as during prolonged periods of sleep deprivation NREM sleep is predominantly restored first. It is important also to point out that according to Deboer, 2018 (49) the amount of sleep lost (NREM or REM sleep) is not totally regained during following hours and/or days and thus the sleep homeostatic 
response does not completely restore the amount of sleep lost during sleep deprivation. Using data from EEG the same author suggests that mammals can compensate sleep loss with two different ways: by increasing the amount of sleep and by increasing the depth of sleep detected by enhanced slow wave amplitude.

The homeostatic mechanism is thought to be due to the accumulation of one or more substances during wakefulness as a result of the increased activity of the nuclei involved in maintaining wakefulness, leading to enhanced need for sleep in order to restore normal brain functions. The underlying mechanisms of these homeostatic determinants however are not completely understood. During prolonged wakefulness an increased hydrolysis of ATP, coming from glucose oxidation probably can lead to an increased accumulation of ADP, AMP and adenosine in extracellular fluid in some brain areas responsible for the arousal drive (22). Adenosine may accumulate extracellularly as a result of cellular metabolism during waking. It is well known that astrocytes are the main energy store in the brain. They contain significant amount of glycogen droplets which are depleted during waking suggesting that astrocytesmay cause an increase in extracellular adenosine, which in turn promotes sleep (50-52). This phenomenon was experimentally demonstrated, indicating that astrocytic adenosine acting through A1 receptors contributes to the modulation of sleep pressure and the regulation of sleep homeostasis following sleep deprivation (5355). As such it has been demonstrated that a non-neuronal cell type of the brain, the astrocytes, modulates sleep behavior. Indeed, elevated extracellular concentration of adenosine in basal forebrain has been found to be associated with increased need for sleep $(53,8)$. Adenosine is a purine nucleoside composed of adenine which is attached to a ribose molecule. Derivatives of adenosine are widely found in nature, including living organisms and play an important role in many biochemical processes, such asenergy transfer as adenosine triphosphate (ATP) and adenosine diphosphate (ADP).

The eventual role of adenosine as one of the important homeostatic factors inducing sleep was further supported by the experimental data, showing that the exogenous microinjection of adenosine and an agonist of adenosine A1 receptor into the basal forebrain in cats or adenosine A2 receptor agonist around VLPO nucleus in rats were able to induce sleep in treated animals. The pivotal role of adenosine A1 receptor in mediating the inhibitory effect of adenosine on cholinergic BF neurons is also confirmed by micro dialysis perfusion of antisense deoxy oligonucleotides (ODNs) against A1 receptor in rats (53). These authors found that micro dialysis perfusion of A1 receptor antisense in the BF caused a marked reduction in non-REM sleep duration, which was accompanied by a significant increase in wakefulness. On the other hands, it has been also hypothesized that adenosine could abolish the inhibitory effect of GABAergic dorsomedial hypothalamic nucleus or noradrenergic input originating from LC in brainstem on VLPO neurons by presynaptic binding to A1 receptors, leading to the activation VLPO nucleus (6). Therefore, based on these observations it can be assumed that adenosine could cause sleep at least in part by reducing the activity of some of the wake provoking nuclei (BF) and/or by activating the sleep promoting neurons (VLPO) (6).

Because apart adenosine, astrocytes release several transmitters it cannot be discount the possibility that additional sleep promoting gliotransmitters may be identified in the near future. In mammalsnitric oxide (NO) and prostaglandin D2 are also reported to be involved in mediating the homeostatic process (2).

\section{Circadian regulation of sleep-wake cycle}

Various physiological functions in the body such as temperature, feeding, hormonal secretions, autonomic activity, sleep-wake cycle etc. are closely associated with changes in daily light/dark time and show fluctuations that occur over a $24 \mathrm{~h}$ period $(22,18)$. These internal $24 \mathrm{~h}$ fluctuations in some physiological functions and behavior are called circadian rhythms and are synchronized and correspond to the external physical environment. Circadian means "about a day". In both humans and diurnal animals the light is thought as the most powerful agent, responsible for the synchronization of circadian rhythms. The suprachiasmatic nucleus (SCN) of the hypothalamus is the brain's internal biological master clock acting as central pacemaker generating circadian rhythms and thus, linking the sleep-wake cycle to daily light-dark periods change (46). The SCN synchronizes the circadian physiological and behavioural 
rhythms, including sleep and wakefulness, temperature, feeding, neuroendocrine and autonomic activities, within a period $24 \mathrm{~h}$ to match the environmental light-dark cycle and thus, establishing an optimal internal cyclic order in numerous important body functions (56, 18;). Actually, there are two suprachyasmatic nuclei located in anterior hypothalamus superior to chyasma optici (22)

The question now is how the light works to promote waking and how the decrease in light intensity promotes sleep? (22). Light excites the photoreceptors in retina and via retinohypothalamic pathway the generated impulses using glutamate as neurotransmitter stimulates SCN (23). It has been established that there is no direct connection between $\mathrm{SCN}$ and VLPO nucleus. SCN affects sleep-wake cycle by indirect connections to VLPO and LH nuclei. There are excitatory output glutamatergic neurons in SCN which project to 2 hypothalamic regions the sub-paraventricular zone (SPZ) and dorsomedial hypothalamus (DMH) $(22,7,9,23)$. The SPZ has 2 distinct parts - dorsal and ventral. Dorsal SPZ is responsible for the circadian rhythm of body temperature. The ventral SPZ projects to the $\mathrm{DMH}$ which is involved in the regulation of sleep-wake cycle in two ways: by sending excitatory glutamatergic projections to $\mathrm{LH}$ and LC and by sending inhibitory GABA-ergic projections to VLPO nucleus $(8,7,9)$. As such the light exerts its wake-promoting effect. In addition, there is an inhibitory pathway from SCN using GABA as neurotransmitter to the hypothalamic paraventricular nucleus (PVN). During the dark period, when this inhibitory pathway is not active, PVN using vasopressin as neurotransmitter stimulates pre-ganglioner sympathetic neurons in intermediolateral column of the lower cervical and upper thoracic part of the spinal cord and thus the outflow to the $m$. dilatator pupilae and the pineal gland $(8,9)$. As a result there is a marked increase in the release of melatonin, which has strong sleep promoting propensity mediated by its stimulation of the inhibitory MT1 receptors on wake-promoting neuronsof the SCN. In this way during the dark period, the wake-promoting region of $\mathrm{SCN}$ is inhibited by the decreased light stimulation from retinohypothalamic pathway and by melatonin, which allows the rapid switch between wake and sleep. The GABA-ergic output neurons of the SCN regulate the circadian changes in the secretion of melatonin - during the day when thece neurons are maximally active as a result of effect of light almost no melatonin is secreted in pineal gland; in contrast, during the night time, when these neurons are not active there is marked increase in the synthesis and secretion of melatonin (9).

\section{Regulation of rapid eye movement sleep (REM sleep) \\ Electroencelographic characteristics of the REM sleep}

The electroencelographic studies in mammals, including humans, indicate the presence of at least 2 main types of brain waves, PGO and theta waves/spikes, generated by different brain structures during REM sleep. This electrical activity is closely associated with the features of REM.

The transition of NREM to REM sleep is initiated by electrical bursts called pontogeniculo-occipital (PGO) spikes or waves originating from some special regions in brain stem reticular formation, lateral geniculate bodies in thalamus and occipital cortex (57; 22). More specifically, the regions in brain stem includes cholinergic neurons known as subcoeruleus - SubC, LDT and PPT areas and glutamatergic neurons, termed sublaterodorsal nucleus - SLD (23). PGO spikes lead to large fluctuations in respiration, thermoregulation, blood circulation, loose of skeletal muscle tone (atonia) and rapid eye movement which do not occur in any other sleeping phase or waking state $(22,2,23,58)$. The PGO spikes are high amplitude-low frequency waves. They are registered only in the period just before the onset of REM sleep, known as transitional REM period lasting about 30-90 s while during the REM sleep itself bursts of low amplitudehigh frequency waves $(2,23)$. It is believed that PGO spikes are source of dreaming periods and visual imagery during REM sleep as they correlate with rapid eye movements and are predominant in visual thalamocortical circuits $(22,2)$. However, the exact neurological network, responsible for the regulation of REM sleep is not yet well understood. The thalamocortical component of the PGO waves is generated in lateral genigulate nucleus (LGN) and subthalamic nucleus and is induced by cholinergic input from LDT/PPT area $(22,2,23)$. The pontine component of PGO waves is generated by noncholinergicbrain stem reticular formation neurons, such as SubC and parabrachial nucleus $(\mathrm{PBN})(2,59,60,58)$. 


\section{Regulation of the transition between non- REM and REM sleep}

Recently, two types of neurons regulating REM sleep and related somatic and behavior changes were found - REM sleep promoting (REM-on) and REM sleep inhibiting (REMoff) as described 22, 9, 23). The REM sleep promoting nuclei are located in lateral hypothalamus (LH), PPT/LTD and in pons sublaterodorsal (SLDN), SubCN and PBN 59; 60). The neurotransmitters used by the neurons in these area are melanin concentrating hormone (LH), acetylcholine (PPT/LDT) and glutamate (SLDN, SubCN and PBN) (22, 7,; 59, 23). REM sleep inhibiting neurons are confined predominantly in brain stem: locus coereleus - LC, dorsal raphe nucleus-DRN and ventrolateralperiaqueductal grey matter (vlPAGM) using as neurotransmitters noradrenaline, serotonin and GABA, respectively $(59,60,23,58)$. The REM sleep promoting neurons are called "REM-on neurons" as they are maximally active during REM sleep and totally inactive during waking and non-REM sleep while the REM sleep inhibiting neurons are called "REM-off neurons" as they are active during waking and non-REM sleep and quiescent during REM sleep $(9 ; 58)$.

An important question is the master control mechanisms of REM sleep. More specifically, the neuronal interactions controlling non-REM - REM sleep transition and alteration are of particular interest. Based on the results from in vitro and in vivo studies in different experimental models the hypothesis for the existence of a "flip-flop" model for switchingin and out of REM sleep similar to that of sleep-wake cycle regulation has been proposed $(7,2,60)$. Indeed, some authors have reported such reciprocal inhibitory links between the REM sleep promoting and REM sleep inhibiting nuclei (2). This model has been described in details in some recent papers $(7,2,59,60,9,58)$. According to this theory during non-REM sleep the REM-off neurons of the brain stem such as serotoninergic neurons in dorsal raphe nucleus, noradrenergic neurons in locus coeruleus and GABA-ergic neurons in ventral periaqueductal grey matter inhibit the REM-on neurons in brain stem cholinergic neurons in PPT/LDT and glutamatergic neurons in sublaterodorsal nucleus, precoerruleus nucleus and parabrachial nucleus. As a result, REM sleep is inhibited. The switch on REM sleep is triggered by melanin concentrating hormone of the lateral hypothalamus neurons which inactivate the REM sleep inhibiting neurons mentioned above - serotoninergic, noradrenergic and GABA-ergic neurons in brain stem and thus disinhibiting (activating) cholinergic in PPT/LDT and glutamatergic (SLD, PC and PBN) REM sleep promoting neurons $(59,60,58)$.

A non-reciprocal transition model has also been evidenced in some studies The involvement of GABA-ergic neurons, nitric oxide, lateral and preoptic hypothalamus in the complex neurological mechanisms regulating the non-REM - REM sleep interactions has been proposed as well (2). It is important to note however, that most of the experimental data in these studies complete and try to elucidate some aspects of the neuronal interactions of the reciprocal model regulating REM sleep.

\section{Regulation of brain cortexactivation during REM sleep}

The general activation of cortex and cortical center of vision (visual cortex) during REM sleep are regulated by different neuronal mechanisms. Cortical activations due to the direct excitatory stimulation by the REM sleep promoting nuclei in brain stem, such as cholinergic PPT/LTD and glutamatergic SLD, $\mathrm{PC}$ and PBN as shown $(2,9,58)$. These authors also reports that visual cortex is activated via an excitatory output from the thalamic lateral geniculate nucleus (LGN), which is stimulated itself by mesopontine cholinergic PPT/LTD nuclei generating the PGO spikes and dreaming during REM sleep.

\section{Regulation of skeletalmuscle atonia during REM sleep}

The disruption of skeletal muscle tonus or atonia in REM sleep is due to the disturbance of the physiological mechanisms of regulation of their activity. It is well known that the activity of striated muscles is stimulated by facilitatory effects of noradrenergic LC on alpha motor neurons in ventral horns of the spinal cord while the inhibition signals are generated in the glycinergic and/or GABAergic neurons of the medullar gigantocellular nucleus (GC) (7). Two mechanisms of the atonia of the skeletal muscles during REM sleep have been suggested - inhibition of the noradrenergic stimulation of motor neurons and glutamatergic activation of glycinergic and 
GABA-ergic neurons in the GC by $\operatorname{SLD}(59,9$, 61, 58). Krenzer et al., 2011 (62) reported a descending nerve fibers from the SLD to the spinal inhibitory interneurons proving the existence of a network between medulla oblongata glutamatergic neurons and spinal interneurons which is implemented in the REM sleep associated skeletal muscles atonia.

\section{Regulation of rapid eye movements during REM sleep}

It is thought that the horizontal conjugate eye movements or rapid eye movements during REM sleep are regulated by the tegmentopontine reticular nucleus (TPR) which activates the nuclei of the III and VI cranial nerves - $n$. oculumotorius and $n$. abducens, respectively (9). The impulses for the activation of TPR nucleus are generated in glutamatergic SLD nucleus in brain stem (9)

\section{Physiological role of melatonin in the regulation of sleep-wake cycle}

As we already described in details, sleep propensity is caused by two processes - the amount of sleep deprivation (homeostatic component) and on the circadian clock phase (circadian component). Both processes work in a coordinated way to cause sleep at night and wakefulness during the day. A close interaction exists between circadian rhythms such as body temperature, blood pressure, immune and hormonal rhythms and the sleepwake cycle leading to optimization of the internal temporal order $(63,66,56)$.

Melatonin is an important physiological factor involved in the regulation of sleep-wake cycle in diurnal species, including humans. Melatonin is high water and lipid soluble, which facilitates it to easy diffuse through membranes in most cell, including the bloodbrain barrier. Its plasma half-life is about 30-40 minutes and is inactivated mostly in the liver and then excreted in the urine as 6sulfatoxymelatonin.

It is important to note that melatonin refers to a circadian clock synchronizer and indirect sleep regulator. As the main function of melatonin is to mediate dark signals and to provide them to the brain and $\mathrm{SCN}$, it is a "hormone of darkness," rather than a hormone of sleep. It has been demonstrated that melatonin does not increase the amount of SWS which is the main marker of the homeostatic sleep pressure $(63,67)$. Therefore, the sleep promoting effects of melatonin are mostly associated with the circadian component of sleep regulation.

It has been found that the pineal gland is involved in the control of many functions of the organism via secretion of melatonin (66). The primary physiological function of melatonin is the regulation and synchronization of biological rhythms in response to the light-dark circadian cycle, including sleep induction (68-73). In addition to this, however, it has also strong antioxidant action and appears to be involved in the regulation of immune reactions and the protection of the organism from carcinogenesis and neurodegenerative disorders, including Alazheimer's and Parkinson diseases (74, 75, 66).

Melatonin is the main hormone secreted by the pineal gland. The biosynthesis of melatonin occurs through a 4 steps pathway starting with its main precursor, the amino acid Ltryptophan: hydroxylation, decarboxylation, acetylation and methylation. In the pineal gland, tryptophan is hydroxylated to 5hydroxy-tryptophan in the mitochondria, then decarboxylated in the pineal cytosol to generate 5-hydroxitryptamine called also as serotonin, another important neurotransmitter. Serotonin can continue to either melatonin synthesis, or participates in other metabolic pathways. For melatonin synthesis, 5hydroxitryptamine is firstly acetylated on its free amine then O-methylated on the hydroxyl group to generate the final product of melatonin.

In invertebrates, melatonin secretion is stimulated by norepinephrine and this effect is mediated by $\beta-1$ adrenergic receptor on pinealocytes (9). This occurs in the evening as a result of the activation of sympathetic neurons in the superior cervical ganglion by the decreased light. Norepinephrine elevates the intracellular cAMP concentration in pinealocytes and activates the cAMPdependent protein kinase A (PKA) which is responsible for the control of the abovementioned steps in melatonin pathway synthesis. On exposure to daylight, noradrenergic stimulation stops and the synthesis of melatonin is almost totally inhibited (9). The synthesis of melatonin starts again in the evening when daily light decreases at the point called the dim-light melatonin 
onset. As such the dim light melatonin onset is a consistent and reliable marker of the intrinsic circadian phase (63).

Melatonin plays a significant role in promoting both the initiation and maintenance of sleep at night (73). This assumption comes from the experimental data, showing that the marked rise in sleep propensity at night normally occurs about $2 \mathrm{~h}$ after the beginning of endogenous melatonin secretion by the pineal gland. On the other hand, the duration of the increased nocturnal melatonin concentration "informs" the brain and the SCN itself about the night (dark) length $(63,56)$.

In most diurnal mammals, including humans, melatonin is secreted at night. The peak plasma concentrations could be detected between 2- $3 \mathrm{~h}$ AM. There is a good correlation between the rise of melatonin secretion and sleep propensity beginning about 2 hours before the regular bedtime. The rhythmic pattern of melatonin release is regulated by the central circadian rhythm generator known also as the internal biological clock - the suprachiasmatic nucleus (SCN) of the anterior hypothalamus. The physiological effects of melatonin are mediated by two membrane receptors: MT1 and MT2 which are members of the G-proteinlinked receptor family (63). Despite the high density of both types in SCN, they are also expressed throughout other brain parts and other organs, such as ovaries, pancreas, cerebral and peripheral arteries, immune cells, indicating that melatonin probably affects function of different physiological systems (66). The phase shifting effects of melatonin are mostly mediated through the MT2 receptor, while the MT1 receptor is more closely related to sleep onset (63).

As we mentioned earlier, two distinct mechanisms of regulation of sleep have been proposed: homeostatic, called process $\mathrm{S}$, and circadian, called process $\mathrm{C}$, respectively. In this respect, it has been suggested that melatonin has two probable interacting effects on the sleep-wake cycle $(63,56)$. First, it advance and shifts the circadian rhythm (process C) in a "chronobiotic" manner. Second, it promotes sleep onset and continuity in a"hypnotic"function by increasing the homeostatic drive to sleep (process $\mathrm{S}$ ).

Using diurnal zebra fish model, Gandhi et al., 2015 (73) have suggested that endogenous melatonin promotes sleep by modulating the internal circadian clock and by directly affecting sleep regulatory network in brain. More specifically, it has been demonstrated that the sleep-promoting effect of melatonin is mainly due to the inhibition of thewakefulness generating nucleiin hypothalamus and SCN in particular $(73,63,56)$. Moreover, the molecular and functional brain imaging studies indicate that exogenous melatonin affects brain network and activity in a manner similar to real sleep in fully awake subjects $(76,63)$.

Actually, the light can exert two main effects on melatonin production: 1. Physiologically, light-dark cycle entrains the circadian pacemaker and synchronizes the rhythm of secretion i.e. increase in the evening and decrease during the day; 2. Acute light exposure at night however, causes a drastic inhibition in melatonin synthesis leading to a rapid fall in melatonin levels in cerebro-spinal fluid and blood (77). A number of recent studies suggest that the chronic exposure to artificial blue light at night (for ex. TV, cell phone, PC) results in a dramatic disruption of the circadian system and internal master clock which has deleterious effect on health and may lead to increased risk for various abnormalities and diseases, such as metabolic syndrome, cardiovascular diseases, diabetes type 2 , cancer $(17,78,79,18,80)$. It is important to point out that these disorders are closely associated with disturbed pineal melatonin secretion.

Melatonin serves as a time cue and conveys signals of darkness toSCN and therefore its phase-shifting effects are opposite to effect of light (56). As a result of its synchronizing effect on the central biological clock, i.e. SCN, melatonin is able to entrain the circadian changes in the body, including sleep-wake cycle, body temperature, hormonal secretion etc. (81, 82). Similarly, exogenous melatonin administration modifies the light effects on circadian phase shifts and can also entrain the circadian rhythms which are mediated by the $\operatorname{SCN}(83,84)$.

Advanced age, neuro-degenerative diseases, diabetic neuropathy, brain tumors, Alzheimer's disease and administration of some drugs i.e. $\beta$-blockers and non steroid anti-inflammatory drugs inhibit melatonin synthesis at night and can cause sleep disorders $(85,63)$. Day-time intake of melatonin when its endogenous rate of production is almost absent induces events 
such as fatigue and sleepiness seen in early night time in response to the physiological dim-light melatonin onset (86-88). The sleeppromoting effects of melatonin appears about 2 hours after exogenous administration which is in line with the physiological sequence at night caused by endogenous melatonin - heat loss, reduced arousal activity and delay in cortisol secretion (89-92). In addition, exogenous melatonin intake induces similar architecture of sleep as during natural sleep detected by EEG (89). Of note, it has been reported that the sleep promoting effect of melatonin is comparable to that of some other hypnotics such as GABA receptor allosteric agonists zopiclone and zolpidem $(88,90)$.

Molecular and functional brain imaging studies reveal some new aspects concerning the effects of exogenous melatonin administration on the activity in some brain areas and especially in the hippocampus $(95,93)$. It has been suggested that melatonin may affect the memory processing and storage as in actual sleep. Some of these patterns resembled those in subjects tested in the late evening, when there is an increased endogenous melatonin secretion $(93,94,63)$. Gorfine et al., 2006 (95) have demonstrated that the mechanism of melatonin induced fatigue on brain activity is markedly different from that after sleep deprivation. These studies indicated that melatonin modulates brain activity patterns in awake subjects in a manner similar to natural sleep (95).

\section{Pharmacology of sleep}

Epidemiological studies have shown that approximately $10 \%$ of the total population in the world has different sleep problems and insomnia in particular, women being about 1.5 times more affected (63). Insomnia is more frequent in adults $(30 \%)$ and reaching $50 \%$ in the over 65 year olds, while the prevalence in $18-35$ year olds is $10-15 \%(63 ; 108)$. The insomnia is a serious health problem often leading to decreased concentration, depression, increased anxiety and general worsening of the well-being. In addition, cognitive functions, memory and immune system can also be disturbed (11-14).

More recently, it has been suggested that chronic insomnia and/or disturbed sleep can lead to increased risk for obesity and related abnormality such as metabolic syndrome, diabetes type 2 and cardio-vascular diseases
(18). In addition, a physiological link between circadian clocks and glucose metabolism has been described, while there is current evidence for a relationship between circadian disruption and insulin resistance (18). Importantly, these authors propose several new strategies for the implementation of chronobiological knowledge with the aim to improve human metabolic health.

The widespread sleep disorders imply the daily intake of different hypnotic medication. The best hypnotic should match at least the following criteria: 1) no effect on memory or cognition; 2) maintenance of physiological sleep; 3) no residual effects; 4) optimal halflife; 5) no potential for abuse, tolerance or dependence.

Hypnotic drugs could be classified in the following groups:

1.Benzodiazepines

2.Z-drugs - cyclopyrrolones

(Zopiclone), imidazopyridines (Zolpidem), pyrazolopyrimidine (Zaleplone)

3.Sedative antidepressants

trazodone, mirtazapine, doxepin

4.Antihistaminergic drugs -

antagonists of type 1 histaminergic receptors

5.Antipsyhotics known also as neuroleptics - Chlorpromazine, Thioridazine, Haloperidol, Droperidol

6.Melatonin, including prolonged melatonin release drugs and melatonin receptor agonists - Agomelatin, Tasimeltieon, Ramelteon

7.Orexin receptors antagonists almorexant, filorexant, suvorexant

Because the sleep promoting effects of benzodiazepines, Z-drugs, antipsyhotics, antihistaminergic drugs and sedative antidepressants are relatively well known, we will pay more attention to some new hypnotic formulations such as prolonged melatonin release drugs, melatonin receptors agonists and orexin receptor antagonists.

The most frequently prescribed medication includes benzodiazipines (short- and intermediate acting) and non-benzodiazepines or Z-drugs - i.e. Zolpidem, Zopiclone, Zaleplon $(64,65)$. The hypnotic effect of both types of drugs is due to their binding and activation of A1 GABA-ergic receptors (allosteric activators) in central nervous system. Benzodiazepines are generally 
effective and safe for short-term use only, lasting no longer than 2-3 weeks. Long-term use of benzodiazipines, however is controversial and not recommended because of many severe side effects concerning decreased effectiveness, tolerability, physical dependence, increased risk of amnesia, dementia and serious withdrawal syndrome when the patients attempt to discontinue the hypnotic drugs (65).

The relatively new classes of hypnotic medication - Z-drugs are non-benzidiazepines type A GABA receptor agonist which works also by increasing GABA effects in brain as benzodiazipines. In addition to some residual "hangover" effects such as sleepiness and disturbance in psychomotor and cognitive functions, the usage of Z-drugs, similar to benzodiazepines, is associated with drug tolerance and substance dependence. That is why they are recommended and prescribed only for sever insomnia and for a short period of use ( $<2$ weeks) at the lowest effective dose $(64,65)$.

It is important to note that benzodiazepines and Z-drugs treat insomnia especially to quantitative aspects of sleep problems i.e. increased sleep latency and decreased total sleep time but none of them affects sleep quality and/or doesn't improves the restorative value of sleep $(63-65 ; 56)$. In addition, their hypnotic mechanism of action is mainly based on activation of GABA effects as allosteric activators of GABA-ergic receptors in the central nervous system, which differs markedly from the naturally occurring sleep. As we already described in details, however, the physiological regulation of the sleep-wake cycle is a result of complex interrelations among nuclei located in different brain areas, using specific neurotransmitters and acting on the basis of the "flip-flop" or "on-off" model in close association with circadian changes in the dark-light period. Furthermore, GABAmimetic medication used at this time (benzodiazepines and non-benzodiazepines Zdrugs) are effective in restoring mainly slow wave non-REM sleep $(4,64,65)$. Almost all of these drugs however, suppress REM sleep and thus do not match completely the physiological mechanisms of normal sleep architectures in humans. Therefore, according to Datta, 2010 (4) the big challenge now to sleep research and sleep medicine is to identify and/or to generate novel pharmacological agents which are able to restore both non-REM and REM sleep.

The main purpose of the therapy of sleep disorders must now change from improving symptoms such as disturbance in initiating and maintaining sleep to re-establishing restorative sleep, i.e. improving sleep quality and efficiency by keeping normal sleep architecture, sleep latency and total sleep time and thus improving daytime functioning (56, $63,65)$.

In this respect, melatonin seems as a good alternative medication to hypnotic drugs due to its well documented synchronizing effect on the central biological clock, i.e. SCN, and its ability to entrain the circadian changes in the body, including sleep-wake cycle. It is well known that the endogenous synthesis of melatonin decreases with age and is usually low in elderly patients suffering from insomnia (56). A number of studies suggest that this is probably due to age related calcification of the pineal gland $(96,63)$. These age-related decline in melatonin levels are often associated with sleep disturbances and worsening of sleep quality (97). In addition, disturbances of SCN circadian activity seen amongst elderly people have also been reported $(97,63,56)$.

According to "melatonin replacement hypothesis" the well established age-related decrease in pineal melatonin production is closely associated with sleep disturbance and poor sleep quality and thus, exogenous melatonin administration may compensate for the reduction in the endogenous melatonin secretion and may improve sleep disorders (98).

The sleep promoting influence of exogenous melatonin in humans however, is not yet fully elucidated. Some important questions concerning age, doses and especially the longterm effects of melatonin administration are still subject of debate. Improvement of sleep in elderly people by high doses of melatonin $(>5$ $\mathrm{mg}$ ) was reported whereas the use of low doses of melatonin didn't show significant effects on sleep in younger insomnia patients $(63,99$, 100). Later the efficacy of lower doses (0.3-5 $\mathrm{mg}$ ) of melatonin in shortening of sleep latency in insomnia elderly patients has been reported $(99,63)$. It is generally accepted that the exogenous melatonin should be taken by patients at the age of 50-55 years and older 
when the pineal gland doesn't function properly and marked decrease in hormone production is observed (99).

Despite these positive effects of exogenous melatonin therapy there are some important limitations. Melatonin is quickly metabolized in liver which results in extremely short plasma half-life - 40-50 min (63). After oral intake, melatonin is rapidly absorbed the peak plasma levels occurring between 20 minutes and 2 hours depending on the dose. Therefore, in order to ensure adequate concentration during the night-time period, very high doses or repeated administration of melatonin is needed. Experimental data in rats and in cell culture suggest, however, that the use of high melatonin doses can causes resistance to the effects of melatonin and desensitizes and inactivates its receptors in SCN (63). Despite no such studies in humans, new formulations of the hormone that would follow the physiological nocturnal increase in melatonin concentrations are needed for melatonin replacement therapy $(99,63,56)$.

A new approach has been recently proposed for melatonin substitution therapy using a prolonged-release formulation (PRM) (99). The idea is to avoid the intake of high doses and/or repeated intake of melatonin during the night. Such formulation would effectively mimic the physiological pattern of nocturnal secretion and profile of the hormone and may be an appropriate alternative to benzodiazepine and non-benzodiazepine treatment of insomnia in older patients $(63,56,100)$. The experimental data from a number of largescale, randomized, placebo-controlled clinical trials in insomnia patients aged 55 years and older, indicate that PRM gave for short (3 weeks) or long term (6 months or 1 year and longer) improves sleep quality, facilitates sleep onset, improves morning alertness and quality of life and as a result reduced the use of hypnotic drugs $(63 ; 56 ; 100)$. In addition, there were none of the severe side effects and risks associated with the use of hypnotic drugs such as memory and cognitive disturbance, residual daytime or 'hangover' effects, rebound insomnia and withdrawal symptoms $(63,56$, 100). Moreover, it has been demonstrated that the sleep promoting effects of PRM are similar to those of the more frequently used hypnotics of the benzodiazepine and non-benzodiazepine groups, i.e. Z-drugs and new generated agonist of M1 and M2 melatonin receptors ramelteon.

Importantly, it has been shown that melatonin potentiates the effects of GABA-A receptor allosteric activators (benzodiazepines and Zdrugs), and thus can be used during the withdrawal period to facilitate the unfavorable symptoms of discontinuation of these drugs $(102,90,63,56)$.

About 10 years ago a PRM formulation Circadin ${ }^{\circledR}$, Neurim Pharmaceuticals, has been authorised by the European Commission on 26 July 2007 for the short-term treatment of primary insomnia and poor quality of sleep in patients who are aged over $55(63 ; 56)$. This is the first melatonin receptor agonist approved for clinical use in the European Union. Circadin ${ }^{\circledR}$ containing $2 \mathrm{mg}$ PRM was designed to provide following intake in the evening about 2 hours before bedtime a melatonin concentration and dynamics in the blood more closely related to the normal physiological release pattern of the hormone (63). This author reports data from a pharmacokinetic study (CMEA. Circadian EPA report, 2007) on melatonin blood concentration profile in patients following intake of the $2 \mathrm{mg}$ dose at 9 PM. The peak melatonin levels in the blood were found 2.6 hours which persisted over 3.5 hours after ingestion and then gradually decreased towards the morning which would correspond to the normal brain melatonin levels at night.

More recently, melatonin receptor agonists were designed and officially recognized for treatment of insomnia - Agomelatin, Tasimeltieon, Ramelteon.These drugs have demonstrated no affinity for the GABA receptors. Miming the physiological effects of melatonin, they work through a different neurochemical pathway. Due to lack of GABA activity they have many great advantages as they have not the potential for abuse or dependence and withdrawal symptoms or rebound insomnia. Agomelatine which is also an antagonist of serotonin 5HT-2C receptors is available as Valdoxane ${ }^{\circledR}$ (Servier), for the treatment of major depression, associated with sleep disorders in adults. Experimental data show that Agomelatine improves sleep and daytime fatigue in patients (103). Tasimelteon $\left(\right.$ HETLIOZ $^{\mathrm{TM}}$ ) is an agonist of the melatonin MT1 and MT2 receptors that has been approved in the USA for the treatment of non- 
24-hoursleep-wake disorder (63, 104). Ramelteon (Rozerem ${ }^{\circledR}$, Takeda), was authorized in USA for the treatment of insomnia especially due to the difficulty in falling asleep $(63,56)$.

Treatment with melatonin and melatonin receptor agonists seems to be a very promising approach, with long-term benefits in sleep disorders because of its ability to increase sleep propensity and to synchronize the internal clock and other internal body rhythms. However, an important question is about the effective dose as in different trials a dose rate between $0.5-10 \mathrm{mg}$ has been reported, without a clear dose-effect relationship. This is probably related to the large inter-individual variability in melatonin bioavailability and endogenous melatonin secretion/level and which may vary 20 fold among healthy adult people (63). In addition, the duration of the treatment period is also under debate. Unfortunately, in addition to these officially authorized melatonin formulations, a big number of uncontrolled melatonin products are however available as food supplements for which there are no studies and thus, no certain data, about their quality, pharmacokinetics or safety. Therefore, further well controlled clinical experiments are warranted to establish the efficacy and safety of melatonin preparations administration in the treatment of different sleep abnormalities.

In addition to melatonin formulations, taking into account some serious adverse effects of most of benzodiazepines and Z-drugs during the last $10-15$ years some new pharmacological agents for treatment of sleep disorders have been designed. In 1998 a novel neuropeptide called hypocretin or orexin was discovered. It is produced by the lateral hypothalamus and has various physiological effects mostly related to the maintaining the wakefulness. Orexinergic neurons constitute an important part of the wake-promoting system via their direct projections to the cortex and some important wake promoting nuclei histaminergic TMN in posterior hypothalamus, noradrenergic LC, dopaminergic VTA, cholinergic lateral dorsal tegmentum/pedunculopontine tegmentum (LDT/PPT) and serotoninergic dorsal raphe nucleus (DR) in brain stem. An important modulator of the synthesis of orexin is ghrelin which is produced by endocrinocytes of stomach mucosa during fasting. Evolutionary, the physiological meaning of this link is that orexin must maintain wakefulness which enables animals to look for food and to avoid dangers. Additional actions of orexinergic system is to stabilize and modulate the rapid transition between sleep and wakefulness known as "flip-flop" switch and the involvement in memory, cognition, attention, emotion, motivation, feeding behaviour and energy homeostasis (105). Two types excitatory orexinergic receptors (OX1R and $\mathrm{OX} 2 \mathrm{R}$ ) are identified which are located mainly in wake promoting nuclei and brain cortex $(106,107,105,108)$. Since that time dual orexin receptor antagonists (DORAs) have been developed such as Almorexant, Filorexant, Suvorexant. Because of some serious side effects especially related to abnormal increase in liver enzyme concentration some of them (Almorexant) is ultimately discontinued while others are still under clinical examinations. Suvorexant (MK4305, Merck),a dual orexin receptor antagonist, isa new class of drugsin development and seems very promising agent for the treatment of insomnia. Suvorexant was approved by the FDA in 2014 for the treatment of insomnia $(107 ; 108)$. Experimental data show that it promotes the natural transition from wakefulness to sleep by inhibiting the wakefulness-promoting orexin neurons of the arousal system $(106,105,108)$. In addition, it has been shown that Suvorexant improves both sleep onset and sleep maintenance and has a favorable tolerability and limited side effects compared to other hypnotic drugs $(107,108)$. Moreover, in contrast to the drugs acting via activating the whole-brain GABA neurons (benzodiazepines and Z-drugs), the scope of orexin signaling in the brain is much more targeted resulting in less adverse reactions (105). Initial trials suggest also that Suvorexant maintains normal sleep architecture as it promotes both NREM and REM sleep, unlike the GABA-mediating agents which are well known to promote NREM-sleep and to inhibit REM-sleep (105). These authors report, that despite these positive effects however, some mild to moderate side effects (somnolence) have been reported for Suvorexant. The available data on the safety of Suvorexant is limited because of the small number of the published studies using mostly healthy volunteers (108). Therefore, more, well controlled clinical trials are needed to better 
evaluate the efficacy and safety of Suvorexant and other DORAs (108).

In conclusion, we can assume that the neurophysiological regulation of sleep comprises complex interactions between homeostatic and circadian mechanisms. At present the "flip-flop" model reflecting reciprocal bidirectional interactions between wake promoting and sleep inducing nuclei in different brain structures seems the best explanation of rapid sleep-wake transition. The most frequently prescribed hypnotic medication includes benzodiazipines (shortand intermediate acting) and nonbenzodiazepines or Z-drugs - i.e. Zolpidem, Zopiclone, Zaleplon. The hypnotic effect of both types of drugs is due to their binding and activation of A GABA-ergic receptors (allosteric activators) in central nervous system. Both classes of drugs however, have too many severe side effects such as decreased effectiveness, tolerability, physical dependence, increased risk of amnesia, dementia and serious withdrawal syndrome when the patients attempt to discontinue the hypnotic drugs. Therefore, the main approach and purpose of the therapy of sleep disorders must be directed to re-establishing restorative sleep, i.e. improving sleep quality and efficiency by keeping normal sleep architecture, sleep latency and total sleep time. In this respect, exogenous melatonin and more specifically, prolonged melatonin release formulations and melatonin receptors agonists seem as a good alternative medication to classical hypnotic drugs due to their initially well documented synchronizing effect on the central biological clock, i.e. SCN, and its ability to entrain the physiological circadian changes in the body, including sleep-wake cycle. In addition, more recently, a new class of hypnotic drugs has been developed known as orexine receptor antagonists. The available data on the safety of these drugs however are still limited because of the small number of the published studies using mostly healthy persons and shorter treatment period. Therefore, additional well controlled clinical trials are needed to better evaluate the efficacy and safety of melatonin formulations and orexine receptor antagonists, especially to test their long-term effects in patients with sleep disorders.

\section{REFERENCES}

1. Nayak, C., Anilkumar, A. EEG Normal Waveforms. In: StatPearls. Treasure Island (FL): StatPearls Publishing, 2019. Available

from: https://www.ncbi.nlm.nih.gov/books/NBK5 39805/

2. Brown, R., Basheer, R., McKenna, J., Strecker, R., McCarley, R. Control of sleep and wakefulness. Psycological Review, 92:1087-1187, 2012.

3. Scammell, T., Arrigoni, E., Lipton, J. Neural circuitry of wakefulness and sleep. Neuron, 93:747-765, 2017.

4. Datta, S. Cellular and chemical neuroscience of mammalian sleep. Sleep Medicine, 11:431-440, 2010.

5. Eban-Rothschild, A., Appelbaum, L., de Lecea, L. Neuronal mechanisms for sleep/wake regulation and modulatory drive. Neuropsychopharmacology, 43:937952, 2018.

6. Saper, C., Scammell, E., Lu, J. Hypothalamic regulation of sleep and circadian rhythms. Nature, 437:1257-1263, 2005.

7. Saper, C., Fuller, P., Pedersen, N., Lu, J., Scammell, E. Sleep state switching. Neuron, 68:1023-1042, 2010.

8. Schwartz, J., Roth, T. Neurophysiology of sleep and wakefulness: basic science and clinical implication. Current Neuropharmacology, 6:367-378, 2008.

9. Szabadi, E. Neuronal networks regulating sleep and arousal: effect of drug. In Drug treatment of sleep disorders, Milestone in drug therapy. A Guglietta (ed.), Springer Internationnal Publishing Switzeland, 2570, 2015.

10.Sexton-Radek, K. A look at worldwide sleep disturbance. Journalof sleep disorders andtherapy, 2:1-3, 2013.

11.Walker, M. P. The Role of Sleep in cognition and emotion. The Year in Cognitive Neuroscience, 1156 (1): 168-197, 2009.

12. Killgore, W. D. S. Effect of sleep deprivation on cognition. Progress in: Brain Research, 185:105-129, 2010.

13.Porkka-Heiskanen, T., Zitting, K. M., Wigren, H. K., Sleep, its regulation and possible mechanisms of sleep disturbances. Acta Physiologica, 208:311-328, 2013.

14.Yaffe, K., Falvey, C. M., Hoang, T. Connections between sleep and cognition in 
older adults. The Lancet Neurology, (13) 10:1017-1028, 2014.

15.Eugene, A., Masiak J. The neuroprotective aspects of sleep. EDtube Sci., 3: 35-40, 2015.

16.Wolk, R., Gami, A., Garcia-Touchard, A., Somers, V. Sleep and cardiovascular disease. Current Problems in Cardiology, 30:625-662, 2005.

17.Maury, E., Ramsey, Bass, J., Circadian rhythms and metabolic syndrome: from experimental genetics to human disease. Circ Res, 106: 447-462, 2010.

18.Stenvers, D. J., Frank, A., Scheer, L., Schrauwen, P., Susanne E. la Fleur, Kalsbeek, A., Circadian clock and insulin resistance. Nature Reviews Endocrinology, 15:75-89, 2019.

19.Tall, A., Jelic, S. How broken sleep promotes cardiovascular disease. Nature, 566:329-330, 2019.

20.Iber, C.. Development of a new manual for characterizing sleep. Sleep, 27:190-202, 2004

21.Silber, M.H., Ancoli-Israel, S., Bonnet, M. H., Chokroverty, S., Grigg-Damberger, M. M., Hirshkowitz, M., Kapen, S., Keenan, S. A., Kryger, M. H., Penzel, T., Pressman, M. R., Iber, C. The visual scoring of sleep in adults. JournalClinical Sleep Medicine, 3:121-131, 2007.

22.Fuller, P., Gooley, J., Saper, C. Neurobiology of the Sleep-Wake Cycle: Sleep Architecture, Circadian Regulation, and Regulatory Feedback. Journal of Biological Rhythms, 21:482-493, 2006.

23.Carley, D., Farabi, S. Physiology of sleep. Spectrum Diabetes Journal 29 (1): 5-8. 2016.

24.Chambon, S., Galtier, M., Arnal, P., Wainrib, G., Gramfort, A. A deep learning architecture for temporal sleep stage classification using multivariate and multimodal time series. IEEE Transactions on Neural Systems and Rehabilitation Engineering, 26 (4): 758-769, 2018.

25.Poe, G. R., Walsh, C. M., Bjorness, T. E., Cognitive neuroscience of sleep. Progress in: Brain Research, 185:1-19, 2010.

26.Fogel, S. M., Smith, C. T.. The function of the sleep spindle:a physiological index of intelligence and a mechanism for sleepdependent memory consolidation. Neuroscience Biobehaviour Review, 35:1154-1165, 2011.
27.Nicolas, M., Lafortune, M., Godbout, J., Barakat, M., Robillard, R., Gaétan Poirier, Bastien, C., Carrier, J. Topography of agerelated changes in sleep spindles. Neurobiology of Aging, 34:468-476, 2013.

28.Fogel, S., Ray, L., Binnie, K., Owen, A. How to become an expert: A new perspective on the role of sleep in the mastery of procedural skills. Neurobiology of Learning and Memory, 125:236-248, 2015.

29.Stone, K. C., Taylor, D. J., McCrae, C. S., Kalsekar, A., Lichstein, K. L. Nonrestorative sleep. Sleep Medicine Review, 12:275-288, 2008.

30.Wilkinson, K., Shapiro, C. Nonrestorative sleep: Symptom or unique diagnostic entity? Sleep Medicine, 13:561-569, 2012.

31.Vyazovskiy, V. , Delogu, A. NREM and REM Sleep: Complementary roles in recovery after wakefulness. Neuroscientist, 20:203-19, 2014.

32. Smith, T., Nixon, M., Nader, R. Posttraining increases in REM sleep intensity implicate REM sleep in memory processing and provide a biological marker of learning potential. Learning and Memory, 11:714-719, 2004.

33. Marshall, L., Born, J. The contribution of sleep to hippocampus-dependent memory consolidation. Trends in Cognitive Sciences, 11(10): 442-450, 2007.

34.Boostani, R., Karimzadeh, F., Nami, M. A comparative review on sleep stage classification methods in patients and healthy individuals. Computer Methods Programs Biomedine, 140:77-91, 2017.

35.Hirshkowiz, M., Schmidt, M. Sleep-related erections: clinical perspectives and neural mechanisms. Sleep Medicine Review, 9:311-329, 2005.

36.Colten, H.R., Altevogt, B.M. Institute of Medicine (US) Committee on Sleep Medicine and Research; Sleep Disorders and Sleep Deprivation: An Unmet Public Health Problem. Colten H.R., Altevogt B.M eds., Washington (DC): National Academies Press (US); 2, Sleep Physiology, 2006. Available from: https://www.ncbi.nlm.nih.gov/books/NBK1 9956/

37.Hobson, J. REM sleep and dreaming: toward a theory of protoconsciousness. Nature Review Neuroscience, 10:803-813, 2009. 
38. Chase, M. Motor control during sleep and wakefulness: clarifying controversies and resolving paradoxes. Sleep Medicine Review, 17:299-312, 2013.

39.Wagner, U., Gais, S., Born, J. Emotional memory formation is enhanced across sleep intervals with high amounts of rapid eye movement sleep. Learning and Memory, 8:112-119, 2001.

40.Genzel, L., Spoormaker, V. I., Konrad, B. $\mathrm{N}$, Dresler, M. The role of rapid eye movement sleep for amygdala-related memory processing. Neurobiologyof learning and memory, 122:110-121, 2015.

41.Lee, M., Katsuyama, Â., Duge, L., Sriram, C., Krushelnytsky, M., Kim, J. J., de la Iglesia, H. Fragmentation of rapid eye movement and nonrapid eye movement sleep without total sleep loss impairs hippocampus-dependent fear memory consolidation. Sleep, 39:2021-2031, 2016.

42.Xia, Z., Storm, D. Role of circadian rhythm and REM sleep for memory consolidation. Neuroscience Research, 118:13-20, 2017.

43.Swihart, B.J., Caffo, B., Bandeen-Roche, K., Punjabi, N.M. Characterizing sleep structure using the hypnogram. Journal of Clinical Sleep Medicine, 4:349-355, 2008.

44.Liu, D., Dan, Y. A motor activity of sleepwake control: arousal-action circuit. Annual Review of Neuroscience, 42:27-46, 2019.

45. Achermann, P., Borbely, A. Mathematical model of sleep regulation. Frontiers in Bioscience, 8:683-693, 2003.

46.Deboer, T. Sleep homeostasis and the circadian clock: Do the circadian pacemaker and the sleep homeostat influence each other's functioning. Neurobiology of Sleep and Circadian Rhythms, 5:68-77, 2018.

47.Panagiotou, M., Meijer, M., Meijer, J.H., Deboer, T. Effects of chronic caffeine consumption on sleep and the sleep electroencephalogram in mice. Journal of Psyhopharmacology, 33:122-131, 2019.

48.Borbély, A., Daan, S., Wirz-Justice, A., et al., The two-process model of sleep regulation: A reappraisal. Journal of Sleep Research, 25:131- 143, 2016.

49.Deboer, T.Behavioral and electrophysiological correlates of sleep and sleep homeostasis.Current Topics in Behavioral Neurosciences, 25:1-24,2015.

50.Petit ,J., Burlet-Godinot, S., Magistretti, P., Allaman,I. Glycogen metabolismand the homeostatic regulation of sleep. Metabolic Brain Diseases, 30:63-79, 2015.

51.Dienel, G., Cruz, N. Contributions of glycogen to astrocytic energetics during brain activation. Metabolic Brain Diseases, 30:281-98, 2015.

52.Dienel, G. Brain Glucose Metabolism: Integration of energetics with function. Physiological Review, 99:949-1045, 2019.

53.Thakkar, M.M., Winston, S., McCarley, R.W. A1 Receptor and Adenosinergic Homeostatic Regulation of SleepWakefulness: Effects of Antisense to the A1 Receptor in the Cholinergic Basal Forebrain. Journal of Neuroscience, , 23 (10): 4278-4287, 2003.

54.Halassa, M., Florian, C., Fellin, T., Munoz, J., Lee, S., Abel, T., Haydon, P., Frank, M. Astrocytic modulation of sleep homeostasis and cognitive consequences of sleep loss. Neuron, 61:213-219, 2009.

55.Hines DJ, Haydon PG. Astrocytic adenosine: from synapses to psychiatric disorders. Phil. Trans. R. Soc. B 369: 20130594, 2014.

56.Zisapel, N. New perspectives on the role of melatonin in human sleep, circadian rhythms and their regulation. British Journal of Pharmacology, 175: 3190-3199, 2018.

57.Mc Carley, R. Mechanisms and models of REM sleep control. Archives Italiennes de Biology, 14: 427-469, 2004.

58.Peever, J., Fuller, P. The biology of REM sleep. Current Biology, 26:1-25-35, 2016.

59.Luppi, P., Clement, O., Sapin, E., Peyron, C., Gervasoni, D., Léger, L., Fort, P. Brainstem mechanisms of paradoxical (REM) sleep generation. Pflugers Archives, 463:43-52, 2012.

60.Luppi, P., Clément, O., Fort, P. Paradoxical (REM) sleep genesis by the brainstem is under hypothalamic control. Current Opinion in Neurobiology, 23:786-92, 2013.

61.Arrigoni, E., Chen, M., Fuller, P. The anatomical, cellular and synaptic basis of motor atonia during rapid eye movement sleep. Journal of Physiology, 19:53915414, 2016.

62.Krenzer, M., Christelle Anaclet, C., Vetrivelan, R., Wang, N., Vong, L., Lowell, B., Fuller, P., Lu, J. Brainstem and spinal cord circuitry regulating REM sleep and muscle atonia. PLOS ONE, 6:1-10, 2011. 
63.Zisapel, N. Melatonin and sleep. The open Neuroendocrinology Journal, 3: 85-95, 2010.

64.Gunja, N. The clinical and forensic toxicology of Z-drugs. J Med Toxicol., 9(2):155-622013, 2013.

65.Brandt, J., Leong, C. Benzodiazepines and Z-Drugs: An updated review of major adverse outcomes reported on in epidemiologic research. Drugs $R \quad D$., 17:493-507, 2017.

66.Kostouglou-Athanassiou, I. Therapeutic applications of melatonin. Therapeutic Advances in Endocrinology and Metabolism, 4:13-24, 2013.

67.Arbon ,EL, Knurowska, M., Dijk, D.J. Randomised clinical trial of the effects of prolonged-release melatonin, temazepam and zolpidem on slow-wave activity during sleep in healthy people. Journal of Psychopharmacology, 29:764-776, 2015.

68.Bjorvatn, B., Pallesen, S. A practical approach to circadian rhythm sleep disorders. Sleep Medicine Reviews, 13:4760, 2009.

69.Cardinali, D., Pagano, E., Scacchi Bernasconi, P., Reynoso, R., Scacchi, P. Disrupted chronobiology of sleep and cytoprotection in obesity: possible therapeutic value of melatonin. Neuro Endocrinological Letters, 32:588-606, 2011.

70.Coogan, A., Thorne, J. Chronotherapeutics and psychiatry: setting the clock to relieve the symptoms. World Journal of Biological Psychiatry, 12(Suppl. 1): 40-43, 2011.

71.Pevet, P., Challet, E. Melatonin: both master clock output and internal time-giver in the circadian clocks network. Journal Physiology-Paris, 105:170-182, 2011.

72.Sánchez-Barceló, E., Mediavilla, M., Tan, D., Reiter, R. Clinical uses of melatonin: evaluation of human trials. Current Medicinal Chemistry, 17:2070-2095, 2011.

73.Gandhi, A., Mosser, E., Oikonomou, G., Prober, D. Melatonin is required for the circadian Regulation of sleep. Neuron, 85:1193-1199, 2015

74.Giannoulia-Karantana, A., Vlachou, A., Polychronopoulou, S., Papassotiriou, I. and Chrousos, G. Melatonin and immunomodulation: connections and potential clinical applications. Neuroimmunomodulation, 13:133-144, 2006.
75.Tan, D., Manchester, L., Terron, M., Flores, L., Reiter, R. One molecule, many derivatives: a never-ending interaction of melatonin with reactive oxygen and nitrogen species? Journal Pineal Research, 42:28-42, 2007.

76.Gorfine, T., Assaf, Y., Goshen-Gottstein, Y., Yeshurun, Y., Zisapel, N. Sleepanticipating effects of melatonin in the human brain. Neuroimage, 31:410-418, 2006.

77.Pandi-Perumal, S. R., Smits, M., Spence, W., Srinivasan, V., Cardinali, D. P., Lowe, A. D., Kayumov, L. Dim light melatonin onset (DLMO):a tool for the analysis of circadian phase in human sleep and chronobiological disorders. Progress in: Neuropsychopharmacology and Biological Psychiatry, 31:1-11, 2007.

78.Cho, Y., Ryu, S. H., Lee, B. R., Kim, K. H., Lee, E., Choi, J. Effectsof artificial light at night onhuman health: A literature review of observational and experimental studies applied to exposure assessment. Chronobiol Int, 32, 1294-310, 2015.

79.Touitou, Y., Reinberg, A., Touitou, D. Association between light at night, melatonin secretion, sleep deprivation, and the internal clock: Health impactsand mechanisms of circadiandisruption. Life Sci, 173:94-106, 2017.

80.Zubidat, A. E., Fares, B., Fares F., Haim, A. Artificial Light at Night of Different Spectral Compositions differentially affects tumor growth in mice: Interaction with melatonin and epigenetic pathways. Cancer Control, 25, 1, 2018.

81.Lewy, A. J., Ahmed, S., Sack, R. L. Phaseshifting the human circadian clock using melatonin. Behav. Brain. Res. 73:131-4, 1996.

82.Arendt, J., Skene, D. J. Melatonin as a chronobiotic. Sleep Med. Rev. 9, 25-39, 2005.

83.McArthur, A. J., Gillette, M. U., Prosser, R. A. Melatonin directly resets the rat suprachiasmatic circadian clock in vitro. Brain. Res, 565:158-61, 1991.

84.Deacon, S., Arendt, J. Addapting to phase shifts II. Effects of melatonin and conflicting light treatment. Physiol. Behav. 59:675-82, 1996.

85.Zisapel, N. Development of a melatonin based formulation for the treatment of insomnia. Drug. Dev, 50: 226-34, 2000. 
86.Dollins, A. B., Zhdanova, I. V., Wurtman, R. J., Lynch, H. J., Deng, M. H. Effect of inducing nocturnal serum melatonin concentrations in daytime on sleep, mood, body temperature, and performance. Proc Natl Acad Sci USA, 91: 1824-1828, 1994.

87.Cajochen, C., Krauchi, K., Wirz-Justice, A. Role of melatonin in the regulation of human circadian rhythms and sleep. $J$ Neuroendocrinol, 15:432-7, 2003.

88.Paul, M. A., Gray, G., Sardana, T. M., Pigeau, R. A. Melatonin and zopiclone as facilitators of early circadian sleep in operational air transport crews. Aviation Space Environ Med, 75:439-443, 2004.

89.Dijk, D. J., Roth, C., Landolt, H. P. Melatonin effect on daytime sleep in men: suppression of EEG low frequency activity and enhancement of spindle frequency activity. Neurosci Lett, 201:13-16, 1995.

90.Wesensten, N. J., Balkin, T. J., Reichardt, R. M., Kautz, M. A., Saviolakis, G. A., Belenky, G. Daytime sleep and performance following a zolpidem and melatonin cocktail. Sleep, 28, 93-103, 2005.

91.Zisapel, N., Tarrasch, R., Laudon, M. The relationship between melatonin and cortisol rhythms: clinical implications of melatonin therapy. Drug Dev Res, 65: 119-225, 2005.

92.Zisapel, N. Sleep and sleep disturbances: biological basis and clinical implications. Cell Mol Life Sci, 64:1174-1186, 2007.

93.Gorfine, T., Zisapel, N. Melatonin and the human hippocampus, a time dependant interplay. J Pineal Res, 43:80-86, 2007.

94.Gorfine, T., Yeshurun, Y., Zisapel, N. Nap and melatonin-induced changes in hippocampal activation and their role in verbal memory consolidation. J. Pineal Res, 43:336-42, 2007.

95.Gorfine, T. Assaf, Y. Goshen-Gottstein, Y., Yeshurun, Y., Nava Zisapel. Sleepanticipating effects of melatonin in the human brain. NeuroImage, 31(1): 410-418, 2006.

96.Mahlberg, R., Kienast, T., Hädel, S., Heidenreich, J., Schmitz, S., Kunz, D. Dieter Kunz Degree of pineal calcification (DOC) is associated with polysomnographic sleep measures in primary insomnia patients. Sleep Medicine, 10:439-445, 2009.

97.Haimov, I., Laudon, M., Zisapel, N., Souroujon, M., Nof, D., Shlitner,A., Herer, P., Tzischinsky, O., Lavie, P. Sleep disorders and melatonin rhythms in elderly people. BMJ Clinical Research, 309:167, 1994

98. Hughes, R., Sack,R., Lewy, A. The role of melatonin and circadian phase in age-related sleep-maintenance insomnia: Assessment in a clinical trial of melatonin replacement. Sleep, 21:52-68, 1998.

99. Wade A., Ford, I., Zisapel N. Nightly treatment of primary insomnia with prolonged release melatonin for 6 months:a randomized placebo controlled trial on age and endogenous melatonin as predictors of efficacy and safety. BMC, 8: 1-18, 2010.

100.Arendt J. Melatonin: Countering chaotic time cues. Front Endocrinol., 10: 1-16, 2019. https://doi.org/10.3389/fendo.2019.00391

101.Ancoli-Israel, S., Martin, J., Jones, D.W., Caligiuri, M., Patterson, T., Harris, M.J., Jeste, D.V. Sleep-disordered breathing and periodic limb movements in sleep in older patients with schizophrenia. Biol Psychiatry., 45(11):1426-32, 1999.

102.Garfinkel, D., Zisapel, N., Wainstein, J., Laudon M. Facilitation of benzodiazepine discontinuation by melatonin. A New Clinical Approach. Archives Internal Medicine, 159, 2456-2460, 1999.

103.Lemoine, P., Guilleminault, C., Alvarez, E. Improvement in subjective sleep in major depressive disorder with a novel antidepressant, agomelatine: randomized, double-blind comparison with venlafaxine. Journal of Clinical Psychiatry, 68: 17231732, 2007

104.Dhillon, S., Clarke, M. Tasimelteon: first global approval. Drugs, 74:505-511, 2014.

105.Janto, K., Roxanne Prichard J., Pusalavidyasagar S.. An update on dual orexin receptor antagonists and their potential role in insomnia therapeutics. Journal of Clinical Sleep Medicine, 14: 1399-1408, 2018.

106.Bennett, T., Bray D., Neville M. Suvorexant, adual orexin receptor antagonist for the management of insomnia. Pharmacy and Therapeutics, 39, 264-266, 2014.

107. Patel, K., Aspesi A., Evoy K. Suvorexant: adual orexin receptor antagonist for the treatment of sleep onset and sleep maintenance insomnia. Ann. Pharmacother., 49: 477-483, 2015.

108. Tampi, RR, Manikkara G, Balachandran S, Taparia P, Hrisko S, Srinivasan S, Tampi DJ. Suvorexant for insomnia in older adults: a perspective review. Drugs in Context, 7: 1-9, 212517. 2018. DOI: $10.7573 /$ dic. 212517 . 